Les corrélations entre les mesures sur l'animal vivant et les caractéristiques de la viande ont été estimées intra-groupe de porcs abattus le même jour. Ces corrélations sont faibles et variables selon la race ou l'échantillon considéré. Aucune corrélation multiple n'est significative entre les mesures sur la viande et les mesures sur le vivant. Des différences significatives existent entre races (dans l'échantillon B) pour la température musculaire, le rythme respiratoire initial, la rétention d'eau et le $\mathrm{pH}$. Les porcs $\mathrm{P} \times \mathbf{P}$ ont les plus faibles températures musculaires, la respiration la plus lente et l'accélération la plus marquée du rythme cardiaque sous l'effet de la chaleur. La qualité de la viande est la plus basse en $\mathrm{P} \times \mathrm{LW}$.

Dans les deux échantillons, des différences significatives existent entre groupes de porcs abattus à des jours différents. Ces différences paraissent plus importantes pour les caractéristiques de la viande que pour les réactions physiologiques de l'animal vivant.

Au vu de ces résultats, il y a peu à espérer d'une sélection sur les critères physiologiques retenus dans cette étude pour améliorer la qualité de la viande chez le porc, sauf peut-être pour les races du type Piétrain. Quoi qu'il en soit, il semble nécessaire d'améliorer la précision des mesures physiologiques et de standardiser les conditions de transport et d'abattage avant de pouvoir apporter une réponse définitive à la question de la sélection du porc vivant sur la qualité de sa viande.

\title{
Physiological, asPects OF MEAT QUALITy AND ADAPTATION IN PIGS (1)
}

David Lister. - U. K. Meat Research Institute, Langford, Bristol BS18 7DY (Great Britain).

Pigs containing significantly increased amounts of lean in their carcasses tend to die more readily at times of stress. Animals surviving to the usual time of slaughter, frequently produce meat of less than optimal quality. This quality cannot be significantly improved by the same modification of slaughter and handling practices from which so-called stress resistant types of pig benefit.

Evidence is presented that mesomorphism in pigs is associated with low resting values for plasma cortisol and lower levels after appropriate adrenal stimulation when compared with less mesomorphic types of pig. Attempts to correct this condition have led to improvements in the quality of the meat when the animals are slaughtered.

A complete explanation of the aetiology of the syndrome still eludes us.

\section{Interaction génotype-alimentation}

\author{
DIE INTERAKTION ZWISCHEN HERDENNIVEAU \\ UND VATER BEI DER MILCHLEISTUNG DES FLECKVIEHS IN BAYERN
}

G. Averdunk und H. Alps. - Bayer. Landesanstalt für Tierzucht, Grub (B. R. D.).

Die Interaktion zwischen Vater und Herdenniveau (3 Stufen : $3650 \mathrm{~kg}, 3650-4 \mathrm{Ig9} \mathrm{kg}$, $4199 \mathrm{~kg}$ ) wurde an einer Stichprobe des Fleckviehs in Bayern untersucht. Das Material beschränkte sich auf Nachkommen von KB-Bullen, die in jeder Stufe wenigstens ro Töchter hatten, sodass 456 Väter mit 68707 Nachkommen berücksichtigt wurden. Die Ergebnisse zeigen, dass die Interaktionen zwar signifikant sind, aber der relative Anteil mit $0.2 \mathrm{I}, 0 . \mathbf{I} 7 \mathrm{bzw}$.

(1) This paper was prepared whilst the author was a visiting research worker at Danish Meta Research Institute, Roskilde, Denmark. 
0.44 p. Ioo für die Milch- und Fettmenge sowie den Fettgehalt gering ist. Da die Interaktion auch durch Unterschiede in der genetischen Variation in den Niveaustufen verursacht sein kann, erfolgte eine Untersuchung der genetischen Parameter in jeder Stufe mit nachstehenden Ergebnissen :

\begin{tabular}{|c|c|c|c|}
\hline & \multicolumn{3}{|c|}{ Heritabilität $\left(h^{2}\right)$} \\
\hline & niedrig & mittel & hoch \\
\hline Milch-kg........ & .210 & .220 & .268 \\
\hline Fett-kg........ & .198 & .200 & .236 \\
\hline Fett- $\% \ldots \ldots \ldots$ & .262 & .287 & .309 \\
\hline
\end{tabular}

Es besteht die Tendenz eines leichten Anstieges der Heritabilität mit steigendem Niveau der Herden bei allen Merkmalen. Die Korrektur der Korrelation zwischen Genotypen unter verschiedenen Umwelten für die Unterschiede in den genetischen Varianzen, Niveaustufen zeigt, dass die Interaktion teilweise hierdurch verursacht ist.

\begin{tabular}{|c|c|c|}
\hline & $\gamma_{\mathrm{G}}$ unkorrigiert & $r_{\mathrm{G}}^{\prime \prime}$ korrigiert \\
\hline Milchmenge $\ldots \ldots \ldots$ & 0.965 & 0.983 \\
\hline Fettmenge $\ldots \ldots \ldots \ldots$ & 0.967 & 0.986 \\
\hline Fettgehalt $\ldots \ldots \ldots \ldots$ & 0.937 & 0.941 \\
\hline
\end{tabular}

Zum gegenwärtigen Stand des Zuchtprogrammes kann daher eine gleiche Rangierung von $\mathrm{KB}-$ Bullen im niedrigen wie hohen Leistungsniveau angenommen werden.

\section{INTERAKTIONEN VON GENOTYP $\times$ MASTMETHODE BEI MAST- UND SCHACHTEIGENSCHAFTEN VON RINDERN}

\section{F. Pirchner, G. Mayrhofer und J. HaAs. - Tierärztliche Hochschule, Wien (Österreich).}

Fraternale Zwillingskälber (27 Paare) wurden nach drei verschiedenen Methoden aufgezogen und gemästet : als Milchmastkälber bis zu drei Monaten, in intensiver Jungstiermast bis zu einem Jahr, als Ochsen mit zweimaliger Alpung bis zu 2 1/4 Jahren. Masse und Gewichte wurden u. a. genommen im Alter von 3 Monaten, $1 / 2$ Jahr und I Jahr. Bei der Schlachtung von allen Tieren (ausgenommen 6 Ochsen der dritten Replikation) wurden neben Ausschlachtungsprozenten die Anteile wertvoller Fleischstücke sowie die chemische Zusammensetzung des $\mathrm{m}$. long. dorsi bestimmt. Alle Werte wurden in Standardabweichungen, gerechnet vom jeweiligen Mittelwert, ausgedrückt. Es wurden die intra-class-Korrelationen für Paare sowie die genetischen Korrelationen zwischen den verschiedenen Eigenschaften in den drei Milieugruppen (Milchmast, intensive Stiermast, Ochsenmast) berechnet.

Von den Paarkorrelationen für Masse und Gewichte waren bei der I/2 Jahresgruppe (1/2 Jahr bei Ochsen und Maststieren, 3 Monate bei Milchmastkälbern) zwei von neun berechneten kleiner als 0.5 , bei der I Jahresgruppe fünf von neun. Bei den Schlachteigenschaften waren 6 von Io Paarkorrelationen kleiner als 0.5 .

Die genetischen Korrelationen waren bei drei von neun Eigenschaften der $\mathrm{I} / 2$ Jahresgruppe kleiner als 0,5 , während es bei der I Jahresgruppe fünf von neun waren. In beiden Gruppen 